\title{
Using post transplant 1 week Tc-99m DTPA renal scan as another method for predicting renal graft failure
}

https://doi.org/10.1515/med-2019-0056

received December 10, 2018; accepted April 29, 2019

\begin{abstract}
Purpose. The aims of this study were to determine whether post transplant renal scan performed at around 1 week can predict graft failure, and to identify the best predictive factors among easy-to-measure variables.
\end{abstract}

Materials and methods. We retrospectively evaluated patients who underwent Tc-99m DTPA renal scan at approximately 1 week after renal transplantation. They were separated into two categories at 3 months after the operation; graft failure and non-failure. Graft failure was confirmed by biopsy (rejection). Non-failure was confirmed either by biopsy or clinical follow-up with serum creatine (Cr). Scan parameters including glomerular filtration rate (GFR), Hilson perfusion index, peaks of the iliac and graft perfusion curves were analyzed. Clinical variables including age, sex, height, weight, systolic blood pressure, serum Cr, type of donated kidney, side of transplant, and immunosuppressant were also analyzed.

Results. Among total 45 patients, graft failure was present in 11 cases. The serum $\mathrm{Cr}$ level was significantly higher in the failure group. Among scan variables, only the GFR was significantly different between groups. GFR of $<44.48 \mathrm{~mL} /$ min was predictive of graft failure (sen $88.9 \%$ ). Serum $\mathrm{Cr}$ level $>2.13 \mathrm{mg} / \mathrm{dL}$ was also predictive (sen $72.7 \%$ ).

Conclusion. GFR on renal scan at approximately 1 week after kidney transplant can predict graft failure.

Keywords: Kidney Transplantation; Radionuclide Imaging; Kidney Function Tests

\footnotetext{
*Corresponding author: Jung-Min Ha, Department of Nuclear Medicine, Chosun University Hospital, 588 Seoseok-dong, dong-gu, Gwangju, Republic of Korea, 501-757, C.P: $+82+1046115140$, Office: +82+622203589, Fax: +82-62+232-9797, Email: jmha@chosun.ac.kr Ari Chong, Department of Nuclear medicine, Chosun University Hospital, Gwangju, Korea

Nam kyu Choi, Min ho Shin, Department of Surgery, Chosun University Hospital, Gwangju, Korea
}

\section{Introduction}

Graft rejection after kidney transplant is a major problem, and a reliable predictor of graft function is necessary for optimal management of renal transplant patients [1]. The current gold standard for the diagnosis of transplant rejection is invasive core needle biopsy, which carries a risk of graft injury and, furthermore, is not immediately feasible in patients who are taking anticoagulants [2]. A non-invasive method to predict rejection after kidney transplant would be ideal.

Renal scintigraphy (scan) is now widely used to evaluate graft function [3]. Technetium-99m (Tc-99m) diethylenetriaminepentaacetic acid (DTPA) is a reliable tracer for renal scintigraphy, as is technetium-99m mercaptoacetyltriglycine (MAG3). Previous studies have revealed good results with scintigraphy in transplanted kidneys. Most of these studies have relied on early scanning, which was performed within 4 days, and even at 1 day, post transplant [3-11], and a comparison of post transplant scans at 3 days and 7 days postoperatively in the same patient seemed to show that only the post transplant day-3 scan could predict the graft outcome [10]. However, for various reasons, early scanning is not always possible, as is the situation at our institution, where renal scans are not routinely performed until approximately 1 week after transplant.

Among the parameters from DTPA scan, Kirchner's index, Hilson's index, the kidney-to-aorta ratio, graft washout, time difference between peak renal perfusion count and peak arterial count, peak renal perfusion count/counts at plateau, peak renal perfusion/peak renal uptake, renal counts at $20 \mathrm{~min} / \mathrm{renal}$ counts at $3 \mathrm{~min}$, and others, may be evaluated to assess graft function [8]. One group has recently reported a new index for predicting graft function based on a formula using existing variables [5], but as yet, there has been no consensus about which indices are most useful $[6,12]$. In addition, in routine clinical practice, the parameters mentioned above are not all easy to obtain. 
An easily measured parameter from renal scintigraphy performed at about 1 week after transplantation is needed. The aims of this study were to find out whether a renal scan performed at approximately 1 week post transplant can be used to predict graft failure and to identify the best predictive factors among easy-to-measure variables.

\section{Materials and methods}

\subsection{Patients}

This retrospective observational study was carried out in accordance with the Declaration of Helsinki. Patients who met all of the following inclusion criteria were enrolled: 1) renal transplantation performed at our institution between the years 2005 to 2010; 2) renal scan performed at approximately 1 week (5 to 8 days) after transplantation; 3) follow-up performed at our institution at approximately 3 months post transplant. We excluded patients whose follow-up data were missing and those whose renal scans were considered incorrect due to failure of DTPA bolus injection. They were categorized into two groups based on the status 3 months after the operation; graft failure group vs non-failure group. Graft failure was confirmed by renal biopsy (rejection). Non-failure group was confirmed either by renal biopsy or by clinical follow-up with normal serum creatinine (Cr) levels $(<1.5 \mathrm{mg} / \mathrm{dL})$ over 1 year. The study methods of patient enrolment and grouping is shown in Figure 1.

\subsection{Renal scintigraphy (Renal scan)}

Patients were instructed to have adequate hydration before scanning. Gamma camera (Dual head SPECT, INFINIA GP3, GE) Tc-99m DTPA (340 MBq intravenous bolus) scans, anterior views, were performed with patients lying in a supine position on the camera bed. The gamma camera was equipped with a low-energy, parallel-hole, all-purpose collimator. Sequential 60-s perfusion images ( $1 \mathrm{~s} /$ frame) and sequential 20-min images (30 s/frame) were obtained. Image processing was performed using Exeleris Functional Imaging Workstation software (GE). Regions-of-interest (ROIs) were defined for the cortex of the transplanted kidney, iliac artery, and background. Each ROI was firstly drawn automatically and then manually corrected by the same technician, and in every case, the same nuclear medicine physician confirmed the ROIs.
Once the ROIs were drawn, the software automatically calculated the glomerular filtration rate (GFR), the Hilson perfusion index [9] $(100 \times$ [area under arterial curve to peak] / [area under renal curve]), time (s) between the peaks of the iliac and graft perfusion curves $(\Delta P)[13]$, and time (s) for the declining counts on the renal perfusion curve to reach half of the peak value ( $\mathrm{T} 1 / 2$ of graft washout; $\left.\mathrm{GW}^{1} \frac{1}{2}\right)[5,13]$ of renal perfusion.

\subsection{Statistical analysis}

\subsubsection{Variables}

Variables from renal scan including GFR, Hilson perfusion index, $\Delta \mathrm{P}$, and $\mathrm{GW} 1 / 2$, were analyzed, along with clinical variables including age, sex, height, weight, diabetes mellitus, underlying renal disease, systolic blood pressure, serum $\mathrm{Cr}$ level at the time of the renal scan, type of donated kidney, side of the transplant, regimen of immunosuppressive therapy, infection, surgical complication and accompanying disease. In addition, we analyzed whether they had an episode of acute kidney injury (AKI) during the 3 months after transplantation (AKI was defined as increase in serum $\mathrm{Cr}$ by $\geq 0.3 \mathrm{mg} / \mathrm{dl}$ within 48 hours or increase in serum $\mathrm{Cr}$ to 1.5 times from baseline which is known or presumed to have occurred within the prior 7 days or urine volume $<0.5 \mathrm{ml} / \mathrm{kg} / \mathrm{h}$ for $6 \mathrm{~h}$ ) [14]. $\mathrm{P}<0.05$ was considered significant.

\subsubsection{Statistical analysis}

We used Shapiro-Wilk normality test to evaluate data for parametric tests including t-test, chi-squared, chisquare for trend, or simple correlation. Data rejected by the normality test were analyzed by non-parametric tests including the Mann-Whitney U test, Fisher's exact test, or Wilcoxon signed-rank test. Receiver-operator curve (ROC) analysis was also performed. MedCalc software (ver. 13.3.3.0; MedCalc, Inc., Ostend, Belgium) was used for the analyses and $p$-values $<0.05$ were considered to be statistically significant.

\subsection{Results}

\subsubsection{Enrolled patients}

As shown in Figure 1, a total of 109 patients underwent renal scan after kidney transplantation at our institution 


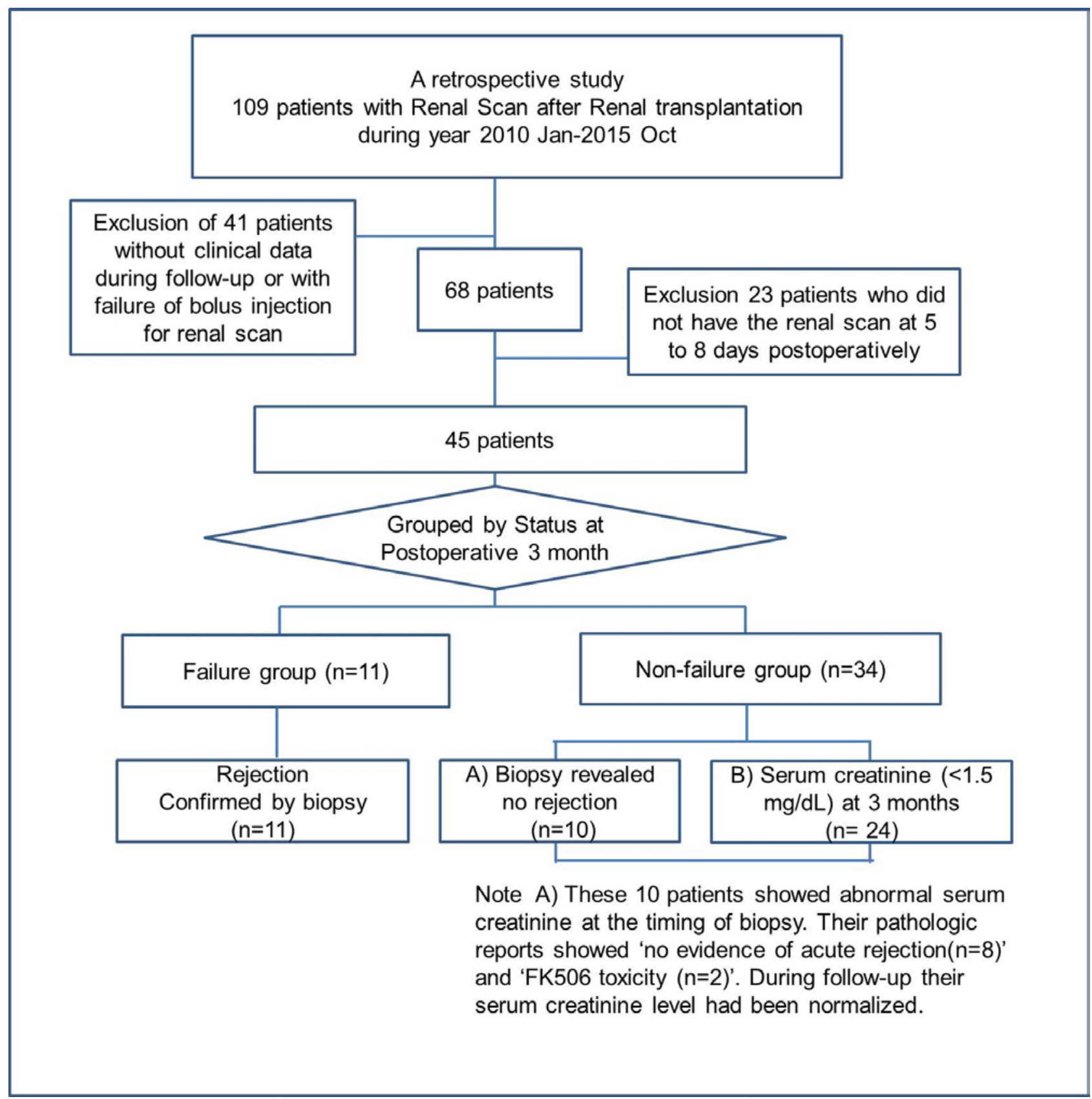

Figure 1: Enrolled patients and grouping

from January 2010 to October 2015. Sixty-four patients were excluded due to lack of clinical data, failure of DTPA bolus injection, or too-long interval between transplantation and scan, and finally, 45 patients who underwent postoperative renal scan at approximately 1 week (day 5 to day 8) after kidney transplant were included.

There were 11 patients who had transplant rejection confirmed by biopsy (Graft failure group). The other 34 patients were in non-failure group. In the non-failure group, 10 patients underwent biopsy and their biopsy results were as follows; 'no evidence of acute rejection $(\mathrm{n}=8)$ ' and 'FK506 (tacrolimus) toxicity $(\mathrm{n}=2)$ '. They under- went renal biopsy due to increased serum $\mathrm{Cr}$ at that time and their serum $\mathrm{Cr}$ level had been normalized later. In all of the patients in the non-failure group, the serum $\mathrm{Cr}$ level remained normal through 1 year post operation. Table 1 shows the patient characteristics for both groups.

Their underlying renal diseases were chronic glomerulonephritis $(n=10)$, hypertensive nephropathy $(n=6)$, diabetic nephropathy $(n=3)$, IgA nephropathy $(n=2)$, chronic interstitial nephritis $(n=1)$, lupus nephritis $(n=1)$, obstructive nephropathy $(n=1)$ and others were unknown origin. Surgical complications were shown in some patients as follows; seroma $(n=4)$, lymphocele $(n=3)$, hematoma $(=1)$, 
Table 1: Patient characteristics at the time of renal scan

\begin{tabular}{ll}
\hline Variable & $27(60 \%)$ \\
\hline Male $^{\mathrm{a}}$ & $45 \pm 10.2$ \\
Age $^{\mathrm{b}}$ & $64.6 \pm 13.7$ \\
Body weight, kg $^{\mathrm{b}}$ & $164.6 \pm 7.8$ \\
Height, $\mathrm{cm}^{\mathrm{b}}$ & $23.71 \pm 4.0$ \\
Body mass index (BMI) $^{\mathrm{b}}$ & \\
Type of donated kidney & \\
DDKT & $38(84.4 \%)$ \\
LDKT $^{\mathrm{a}}$ & $4(8.9 \%)$ \\
ABOi LDKT & $3(6.7 \%)$ \\
Side of transplant, right & \\
Time from operation to renal & $1.27 \pm 0.87$ months \\
biopsy, & $(0.3$ to 3.0 months $)$ \\
Time from operation to DTPA scan & $7 \pm 0.89$ days (5 to 8 days) \\
\hline
\end{tabular}

a $\mathrm{n}(\%)$

${ }^{\mathrm{b}}$ mean \pm standard deviation

' Renal biopsy was performed in 21 patients

Abbreviations: scan, scintigraphy; DDKT, deceased donor kidney transplant; LDKT, living donor kidney transplant; ABOi LDKT, ABO-incompatible LDKT.

leakage $(\mathrm{n}=1)$, hydronephrosis $(\mathrm{n}=1)$, and wound infection $(n=1)$. Accompanying disease before kidney transplantation were shown in some patients as follows; chronic hepatitis $C(n=2)$, dilated cardiomyopathy $(n=2)$, gout $(n=3)$, hepatitis $B$ virus carrier $(n=1)$, hypothyroidism $(n=1)$, overlap syndrome of rheumatoid arthritis and systemic lupus erythematosus $(n=1)$, thyroid cancer $(n=2)$, Crohn's disease $(n=1)$.

Infection during follow-up were shown in some patients as follows; cytomegalovirus infection $(n=1)$, pulmonary fungal infection $(n=1)$, influenza $(n=1)$, MRSA $(\mathrm{n}=1)$, VRE $(\mathrm{n}=1)$, and suprapubic abscess $(\mathrm{n}=1)$.

\subsubsection{Comparison between groups}

Table 2 shows the comparison of results between groups. Among clinical variables, serum $\mathrm{Cr}$ level at the time of renal scan was significantly higher in the Graft failure group, and type of kidney donation was also significantly different $(p=0.0208)$ between groups. The extended chi- square test showed significantly different ratios of graft failure between donor types, and ABO-incompatible living donor showed a higher level of graft failure. The other clinical variables were not different between groups (Table 2).

Underlying renal disease was not significantly different between groups (chi-squared test, $\mathrm{p}=0.0601$ ). Surgical complication was not significantly different between groups (chi-squared test, $\mathrm{p}=0.2192$ ). Accompanying disease and infection were not significantly different between groups either.

Among renal scan variables, GFR was significantly different between groups, while there were no significant between-group differences in other renal scan variables. In two cases of failure group (rejection), the renal uptake was too low. The program could not calculate GFR. Therefore, GFR of failure group was calculated with the scan of 9 patients.

Table 3 shows that GFR of $<44.48 \mathrm{~mL} / \mathrm{min}$ at 1 -week renal scintigraphy post operation could predict graft failure within 3 months (sensitivity 88.9\%, specificity 64.7\%; AUC 0.724; $\mathrm{p}=0.0067)$; among the clinical variables, serum Cr level of $>2.13 \mathrm{mg} / \mathrm{dL}$ also predicted graft failure (sensitivity $72.7 \%$, specificity $82.4 \%$; AUC, 0.734 ; $\mathrm{p}=0.0158)$. Predictive value of both criteria together (serum $\mathrm{Cr}>2.13 \mathrm{mg} / \mathrm{dL}$ and GFR $<44.48 \mathrm{~mL} / \mathrm{min}$ ) and of at least one criteria (serum $\mathrm{Cr}>2.13 \mathrm{mg} / \mathrm{dL}$ or GFR $<44.48 \mathrm{~mL} /$ min) were analyzed, and AUC values were highest for "at least one criteria positive" (0.753), although there was no significant difference between scenarios.

\section{Discussion}

Our results indicate that renal scan performed within 5 to 8 days after kidney transplant could predict graft failure. GFR $<44.48 \mathrm{~mL} / \mathrm{min}$ on renal scan can predict graft failure. Also, when it was impossible to get automatically calculated GFR due to too-low renal uptake, both of those two cases were all in failure group in our study. We also found that serum $\mathrm{Cr}$ level of $>2.13 \mathrm{mg} / \mathrm{dL}$ predicted graft failure. Transplant recipients who meet either or both of these criteria should be closely monitored. Our result is well-correlated with previous studies that mention the importance of GFR as a prognostic factor in renal transplantation[15], and our study confirms that GFR measured by renal scan at approximately 1 week after transplantation can predict the graft outcome.

As mentioned above, in a previous study comparing post transplant on day-3 and day-7 scans, postoperative 
Table 2: Comparison between groups

\begin{tabular}{|c|c|c|c|}
\hline & Non-failure group $(n=34)$ & Graft failure group $(n=11)$ & P-value \\
\hline \multicolumn{4}{|l|}{ Clinical variables } \\
\hline Age $^{a}$ & $45.4 \pm 9.9$ & $45.2 \pm 11.2$ & $0.9489^{b}$ \\
\hline Height ${ }^{\mathrm{a}}$ & $163.0 \pm 7.3$ & $168.0 \pm 6.1$ & $0.0546^{\mathrm{b}}$ \\
\hline Weight $^{\mathrm{a}}$ & $63.9 \pm 14.7$ & $66.8 \pm 10.3$ & $0.5453^{b}$ \\
\hline Body mass index (BMI) ${ }^{a}$ & $23.9 \pm 4.4$ & $23.2 \pm 2.5$ & $0.6251^{\mathrm{b}}$ \\
\hline Diabetes mellitus (yes:no) & $9: 25$ & $6: 5$ & $0.0896^{c}$ \\
\hline Hypertension (yes:no) & $27: 7$ & $10: 1$ & $0.6568^{\mathrm{d}}$ \\
\hline $\begin{array}{l}\text { Serum creatinine }(\mathrm{mg} / \mathrm{dL}) \\
\text { at the time of renal scintigraphye }\end{array}$ & $1.58(0.55-5.886)$ & $3(1.1-13.3)$ & $0.0208^{f}$ \\
\hline $\begin{array}{l}\text { Systolic blood pressure }(\mathrm{mmHg}) \\
\text { at the time of renal scintigraphy }\end{array}$ & $\begin{array}{l}135 \\
(120-180)\end{array}$ & $\begin{array}{l}140 \\
(120-160)\end{array}$ & $0.2909^{f}$ \\
\hline $\begin{array}{l}\text { Type of donated kidneys } \\
\text { (DDKT, ABOi-LDKT, LDKT) }\end{array}$ & $32,0,2$ & $6,3,2$ & $0.0022^{c}$ \\
\hline Right-sided transplant ${ }^{\mathrm{g}}$ & 33 & 10 & $0.4333^{d}$ \\
\hline Episode of acute kidney injury(AKI) (yes:no) & $7: 27$ & $5: 6$ & $0.1089^{c}$ \\
\hline Regimen of immunosuppressant & & & $0.6568^{d}$ \\
\hline Cyclosporine, MMF, PDS & 7 & 1 & \\
\hline Tacrolimus, MMF, PDS & 27 & 10 & \\
\hline \multicolumn{4}{|l|}{ Renal scan variables } \\
\hline $\mathrm{GFR}^{\mathrm{e}, \mathrm{i}}$ & $54.3(14.2-178.4)$ & $34.2(19.1-78.6)$ & $0.0394^{f}$ \\
\hline Hilson perfusion Index & $130.5(24.7-594.1)$ & $141.4(32.0-1002.5)$ & $0.6369^{f}$ \\
\hline$\Delta \mathrm{P}^{\mathrm{e}}$ & $2.06(1.09-4.48)$ & $1.74(1.13-4.48)$ & $0.2259^{f}$ \\
\hline GW $1 / 2$ & $8.5(3.5-93.81)$ & $16.75(3.33-88.48)$ & $0.6446^{f}$ \\
\hline
\end{tabular}

\footnotetext{
${ }^{\text {a }}$ mean \pm standard deviation

${ }^{\mathrm{b}} \mathrm{T}$-test

${ }^{c}$ Chi-square test

d Fisher's exact test

e median (range, minimum to maximum)

${ }^{\mathrm{f}}$ Mann-Whitney test

${ }^{g}$ Number of cases

${ }^{\mathrm{h}}$ Episode of AKI during 3 months after transplantation (AKI was defined as increase in serum $\mathrm{Cr}$ by $\geq 0.3 \mathrm{mg} / \mathrm{dl}$ within 48 hours or increase in serum $\mathrm{Cr}$ to 1.5 times from baseline which is known or presumed to have occurred within the prior 7 days or urine volume $<0.5 \mathrm{ml} / \mathrm{kg} / \mathrm{h}$ for $6 \mathrm{~h} \mathrm{[14]).}$

${ }^{i}$ In two cases of failure group, the renal uptake was too low. The program could not calculate GFR. Therefore, GFR of failure group was calculated with the scan of 9 patients.

Abbreviations: BMI, body mass index; DDKT, deceased donor kidney transplant; LDKT, living donor kidney transplant; ABOi-LDKT, ABO-incompatible LDKT; GFR, glomerular filtration rate; $\triangle \mathrm{P}$, time (s) between the peaks of the iliac and graft perfusion curves; GW $1 / 2$, time (s) for declining counts on the renal perfusion curve to reach half of the peak value ( $T 1 \frac{1}{2}$ of graft washout); MMF, Mycophenolate mofetil; PDS, prednisolone
} 
Table 3: ROC analysis to predict graft failure

\begin{tabular}{lllll}
\hline Variable & AUC & SE & $95 \% \mathrm{Cl}$ & $\mathrm{p}$ \\
\hline Serum Cr & & 0.581 to 0.854 & 0.0158 \\
\hline GFR $^{\mathrm{b}}$ & 0.734 & 0.0970 & 0.568 to 0.850 & 0.0067 \\
\hline Positive both criteriac $^{\mathrm{c}}$ & 0.725 & 0.0832 & 0.577 to 0.083 & 0.0056 \\
\hline Positive at least one criteria $^{\mathrm{d}}$ & 0.763 & 0.0830 & 0.613 to 0.877 & $<0.0001$ \\
\hline
\end{tabular}

Foot note

a, Serum $\mathrm{Cr}$ at the time of renal scan $>2.13 \mathrm{ng} / \mathrm{dL}$

b, GFR on renal scan $<44.48 \mathrm{~mL} / \mathrm{min}(\mathrm{n}=43)$

In two cases, renal uptake was so low that GFR could not be calculated.

c, serum $\mathrm{Cr}>2.13 \mathrm{ng} / \mathrm{dL}$ AND GFR $<44.48 \mathrm{~mL} / \mathrm{min}$

d, serum $\mathrm{Cr}>2.13 \mathrm{ng} / \mathrm{dL}$ or GFR<44.48 mL/min

Abbreviation: AUC, area under curve; SE, standard error; $\mathrm{Cl}$, confidence interval

day-3 scans could predict graft failure, while the day-7 scans could not [10]. That study defined graft failure by long term follow-up (average follow-up duration approximately 67.4 months), whereas the follow-up to graft failure in our study (approximately 3 months) was much shorter. In addition, the previous study used a MAG3 tracer, but it has been reported that DTPA is more sensitive than MAG3 for diagnosing dysfunction [16]. These differences of follow-up period and tracer might have caused the different results, and additional study with a larger number of patients and longer follow-up is needed.

Other variables from renal scintigraphy, including the Hilson perfusion index, $\Delta \mathrm{P}$, and $\mathrm{GW} 1 / 2$, did not predict graft failure in our study. Yazici et al. have reported that patients whose grafts were failing at 3 months after transplant had high $\mathrm{GW}^{1} 1 / 2$ or high $\Delta \mathrm{P}$ on renal scans performed within 2 days after transplant [6]. Those authors determined graft function by serum Cr levels. GW $1 / 2$ and $\Delta \mathrm{P}$ were also high in the failure group in our study, but the finding was not statistically significant. Gupta SK et al. have also reported that among several variables, only Hilson's perfusion index proved to be useful for correlation with pathologic results [7]. However, the goal was correlation between renal scan and pathologic results. Therefore, in their study, most of the enrolled patients underwent biopsy, and the interval between renal scan and biopsy was short (within five days). In our study, the goal was to predict graft failure and not to compare the results of examinations which were done at almost the same time.
In analyzing renal function by scintigraphy, it is very important to draw the ROI in the right place [17]. A purely automated definition of the ROI cannot accurately identify the boundaries of the kidneys [18]. We used a combination of automatic and manual methods, and the same technician drew the ROIs, all of which were confirmed by a nuclear medicine physician.

Three of the patients who had ABO-incompatible living donor transplants had graft failure in this study. Due to the small number of cases, we did not analyze this type of transplant separately. Once again, further study with a larger number of cases and more information will be needed.

There are several limitations in this study. This is a retrospective study. In some cases, clinical information, such as the need for dialysis in the first week, was not available. Delayed graft function is typically defined in patients who need dialysis in the first week after transplant, and lacking those clinical data, we could not distinguish delayed graft function in our analysis. Renal scans were performed 5 to 8 days after transplantation, so patients with delayed graft function might have been included among the patients who were categorized as non-failure group in our analysis. However, in all of the patients in the non-failure group, the serum $\mathrm{Cr}$ level remained normal during the 1st year post operation which means our "non-failure group" was really "non-failure" and remained "non-failure" for a year. Acute tubular necrosis (ATN), also known as acute vasomotor nephropathy, is a common cause of delayed graft function [5, 17], but the presence of ATN was not analyzed in our study because of the timing ( 3 months post 
transplant) of the renal biopsy. Patients with other complications, including cyclosporin toxicity, were also categorized as non-failure group because of these limitations. The small study population is also a limitation.

\section{Conclusion}

DTPA renal scan performed at approximately 1 week after renal transplantation can predict graft outcome at 3 months. Lower GFR can be used to predict graft failure at 3 months.

Acknowledgement: This study was supported by the Chosun University research fund (2017).

Conflict of Interest: The authors declare that they have no conflicts of interest.

\section{Reference}

[1] Santos, J. and L.S. Martins, Estimating glomerular filtration rate in kidney transplantation: Still searching for the best marker. World Journal of Nephrology, 2015. 4(3): p. 345-353

[2] Thölking, G., et al., Imaging-based diagnosis of acute renal allograft rejection. World Journal of Transplantation, 2016. 6(1): p. 174-182

[3] Benjamens, S., et al., Can transplant renal scintigraphy predict the duration of delayed graft function? A dual center retrospective study. PloS One, 2018. 13(3): p. e0193791

[4] Barba, J.F., et al., [Study on the findings of an immediate renal gammagraphy and its effect on the survival of a kidney graft]. Actas Urologicas Espanolas, 2011. 35(4): p. 218-224

[5] Yazici, B., et al., A New Quantitative Index for Baseline Renal Transplant Scintigraphy With 99mTc-DTPA in Evaluation of Delayed Graft Function and Prediction of 1-Year Graft Function. Clinical Nuclear Medicine, 2016. 41(3): p. 182-188

[6] Yazici, B., et al., Evaluation of Renal Transplant Scintigraphy and Resistance Index Performed Within 2 Days After Transplantation in Predicting Long-Term Graft Function. Clinical Nuclear Medicine, 2015. 40(7): p. 548-552
[7] Gupta, S.K., et al., Quantitative (99m)Tc DTPA renal transplant scintigraphic parameters: assessment of interobserver agreement and correlation with graft pathologies. American Journal of Nuclear Medicine and Molecular Imaging, 2014. 4(3): p. 213-224

[8] Gupta, S.K., et al., Quantitative Tc-99m DTPA renal transplant scintigraphy predicts graft survival in the very early postoperative period. Nuclear Medicine Communications, 2012. 33(12): p. 1292-1299

[9] Hilson, A.J., et al., Dynamic renal transplant imaging with Tc-99m DTPA ( $\mathrm{Sn}$ ) supplemented by a transplant perfusion index in the management of renal transplants. Journal of Nuclear Medicine: Official Publication, Society of Nuclear Medicine, 1978. 19(9): p. 994-1000

[10] Park, U.-J., et al., Use of early postoperative MAG3 renal scan to predict long-term outcomes of renal transplants. Experimental and Clinical Transplantation: Official Journal of the Middle East Society for Organ Transplantation, 2013. 11(2): p. 118-121

[11] Gençoğlu, E.A., et al., Scintigraphic evaluation of paired renal allografts from the same cadaveric donor. Transplantation Proceedings, 2004. 36(1): p. 95-98

[12] Ell, P.J. and S.S. Gambhir, Nuclear medicine in clinical diagnosis and treatment. 3rd ed ed. 2004: Edinburgh ; New York: Churchill Livingstone

[13] Preston, D.F. and R.G. Luke, Radionuclide Evaluation of Renal Transplants. Journal of Nuclear Medicine, 1979. 20(10): p. 1095-1097

[14] Nagarajan, M., et al., Impact of acute kidney injury on renal allograft survival. Ren Fail, 2017. 39(1): p. 40-44

[15] First, M.R., Renal function as a predictor of long-term graft survival in renal transplant patients. Nephrology, Dialysis, Transplantation: Official Publication of the European Dialysis and Transplant Association - European Renal Association, 2003. 18 Suppl 1: p. i3-6

[16] Aktaş, A., et al., Comparison of Tc-99m DTPA and Tc-99m MAG3 perfusion time-activity curves in patients with renal allograft dysfunction. Transplantation Proceedings, 2006. 38(2): p. 449-453

[17] Kampa, N., et al., Effect of region of interest selection and uptake measurement on glomerular filtration rate measured by 99 mTc-DTPA scintigraphy in dogs. Veterinary Radiology \& Ultrasound: The Official Journal of the American College of Veterinary Radiology and the International Veterinary Radiology Association, 2002. 43(4): p. 383-391

[18] Tian, C., et al., A semi-automated region of interest detection method in the scintigraphic glomerular filtration rate determination for patients with abnormal low renal function. Clinical Nuclear Medicine, 2013. 38(11): p. 855-862 